\title{
Design of Two-Dimensional IIR Filters Using an Improved DE Algorithm
}

\author{
Swagatam Das ${ }^{1}$ and Debangshu Dey ${ }^{2}$ \\ ${ }^{1}$ Dept. of Electronics and Telecommunication Engineering \\ ${ }^{2}$ Dept. of Electrical Engineering, \\ Jadavpur University, \\ Kolkata-700032, India \\ swagatamdas19@yahoo.co.in
}

\begin{abstract}
The paper investigates a novel technique of designing 2-dimensional IIR digital filters using a modified version of Differential Evolution (DE) where the scalar factor used for weighing the difference vector is made to vary randomly. This approach makes the classical DE more stochastic and provides it with additional exploration capability over the search space. The task of the design has been reformulated as a constrained minimization problem and is solved by the convergence of the proposed algorithm. Numerical example has been provided in support of the theoretical results. The paper also attempts to demonstrate the superiority of the proposed design method by comparing with a few previous design methods based on GA and neural networks.
\end{abstract}

\section{Introduction}

Two-dimensional zero-phase digital filters find an extensive application in the domain of digital image processing, biomedical imaging and digital mammography, X-rays image enhancement, seismic data processing etc [1]-[3]. The most popular design methods for 2-D IIR filters are either based on an appropriate transformation of 1D filter [2], [3]; or based on appropriate optimization techniques. However one of the major problems underlying the design task is to satisfy the stability criterion for the filter transfer function. Although researchers have attempted to tackle the stability problem in a number of ways, most of these efforts resulted into a filter usually having very small stability margin with hardly any practical importance [4]. In this work the design task of 2D recursive filter has been formulated as a constrained optimization problem and an improved DE scheme is applied to solve the same. Numerical results show that [7] the DE-RANDSF (DE with Random Scale Factor) algorithm used here yields a better approximation of the transfer function as compared to works presented in [4] and [5].The proposed technique also satisfies the stability criterion which has been presented as constraints to the minimization problem. Compared to GA based method, the proposed algorithm is easy to implement and takes lesser number of error function evaluations to find an acceptable solution. 


\section{Problem Formulation}

Let the general prototype 2-D transfer function for the digital filter be

$$
H\left(z_{1}, z_{2}\right)=H_{0} \frac{\sum_{i=0}^{N} \sum_{j=0}^{N} p_{i j} z^{i} z^{j}}{\prod_{k=1}^{N}\left(1+q_{k} z_{1}+r_{k} z_{2}+s_{k} z_{1} \cdot z_{2}\right)}
$$

with $\mathrm{P}_{00}=1$ always. Also let us assume that the user specified amplitude response of the filter to be designed is $\mathrm{M}_{\mathrm{d}}$ which is obviously a function of digital frequencies $\omega_{1}$ and $\omega_{2},\left(\omega_{1}, \omega_{2} \in[0, \pi]\right)$. Now the main design problem is to determine the coefficients in the numerator and denominator of (1) in such a fashion that $H\left(Z_{1}=e^{j \omega_{1}}, Z_{2}=e^{j \omega_{2}}\right)$ follows the desired response $\mathrm{M}_{\mathrm{d}}\left(\omega_{1}, \omega_{2}\right)$ as closely as possible. Such approximation of the desired response can be achieved by minimizing,

$$
J\left(p_{i j}, q_{k}, r_{k}, s_{k}, H_{0}\right)=\sum_{n_{1}=0 n_{2}=0}^{N_{1}} \sum^{N_{2}}\left[\left|M\left(\omega_{1}, \omega_{2}\right)\right|-M_{d}\left(\omega_{1}, \omega_{2}\right)\right]^{b}
$$

where

$$
M\left(\omega_{1}, \omega_{2}\right)=\left.H\left(z_{1}, z_{2}\right)\right|_{\substack{z_{1}=e^{j \omega_{1}} \\ z_{2}=e^{j \omega_{2}}}}
$$

where

$$
\begin{aligned}
& \omega_{1}=\left(\pi / N_{1}\right) n_{1} ; \\
& \omega_{2}=\left(\pi / N_{2}\right) n_{2} ;
\end{aligned}
$$

and $b$ is an even positive integer (usually $b=2$ or $b=4$ ).

Here the prime objective is to reduce the difference between the desired and actual amplitude response of the filter at $\mathrm{N}_{1} \cdot \mathrm{N}_{2}$ points. Since the denominator contains only first degree factors, we can assert the stability conditions following [1]-[3] as

$$
\left|q_{k}+r_{k}\right|-1<s_{k}<1-\left|q_{k}-r_{k}\right|, \quad \mathrm{k}=1,2 \ldots \mathrm{N}
$$

Thus the design of a 2-D recursive filter is equivalent to the following constrained minimization problem:

Minimize $J$

$$
\left.=\sum_{n_{1}=0}^{N_{1}} \sum_{n_{2}=0}^{N_{2}}|| M\left(\frac{\pi n_{1}}{N_{1}}, \frac{\pi n_{2}}{N_{2}}\right) \mid-M_{d}\left(\frac{\pi n_{1}}{N_{1}}, \frac{\pi n_{2}}{N_{2}}\right)\right]^{b}
$$

subjected to the constraints imposed by (4).

In [4] the design problem has been tackled with neural networks and [5] attempts to solve it using GA. In the present work a better solution has been presented using a modified version of Differential Evolution (DE) algorithm. 


\section{A Brief Overview of Modified DE Algorithm}

Classical DE [6] searches for a global optimum point in an $\mathrm{N}$-dimensional hyperspace. It begins with a randomly initialized population of N-dimensional realvalued parameter vectors. Each vector forms a candidate solution to the multidimensional optimization problem. Unlike the conventional GA, the reproduction scheme in DE is maintained as follows. For each individual vector $G_{k}{ }^{D}$ belonging to generation D, randomly sample three other individuals $G_{i}^{D}, G_{j}^{D}$ and $G_{m}{ }^{D}$ from the same generation (for distinct $i, j, k$ and $m$ ), calculate the difference of the components (chromosomes) of $G_{i}{ }^{D}$ and $G_{o}{ }^{d}$, scale it by a scalar $\mathrm{R}(\in[0,1])$ and create a trial vector by adding the result to the chromosomes of $G_{k}{ }^{D}$.

$$
\left.\begin{array}{rlrl}
G_{k, n}{ }^{D+1} & =G_{m, n}{ }^{D}+R \cdot\left(G_{i, n}{ }^{D}-G_{j, n}{ }^{D}\right) & & \text { if } \operatorname{rand}_{\mathrm{n}}(0,1)<\mathrm{CR} \\
& =\mathrm{G}_{\mathrm{k}, \mathrm{n}} \mathrm{D}, & & \text { otherwise. }
\end{array}\right\}
$$

for the $\mathrm{n}$-th component of each parameter vector.

$\mathrm{CR}(\in[0,1])$ is the crossover constant. Parameters $\mathrm{R}$ and $\mathrm{CR}$ govern the convergence speed and robustness of DE. The trial solution is evaluated and replaces its parent $G_{k}{ }^{D}$. deterministically if its fitness is better. This alteration and selection procedure is also known as the 'DE/rand/1/bin' operator. There are also other frequently used DE operators, such as 'DE/rand/1/bin' and'DE/best/2/exp', which we did not investigate in this study.

In the original DE the difference vector $\left(\mathrm{G}_{\mathrm{i}}-\mathrm{G}_{\mathrm{j}}\right)$ is scaled by a constant factor ' $R$ '. Usually the most popular choice for this control parameter in $\mathrm{DE}$ is in the range of $(0.4,1)$. However we set this scale factor to change in a random manner in the range $(0.5,1)$ by using the relation

$$
\mathrm{R}=0.5 *(1+\operatorname{rand}(0,1))
$$

where rand $(0,1)$ is a uniformly distributed random number within the range $(0,1)$. The mean value of the scale factor remains at 0.75 . This allows for stochastic variation in the amplification of the difference vector and hence helps retain the population diversity as the search progresses. This new version of DE will be hereafter referred as DE-RANDSF ((DE with Random Scale Factor) algorithm.

\section{Application of the Algorithm to the Design Problem}

\subsection{Converting the Problem to a Soluble Form}

Without loss of generality let us assume $\mathrm{N}=2$ in equation (1). Now if we substitute $Z_{1}$ and $Z_{2}$ as in (3), then $M\left(\omega_{1}, \omega_{2}\right)$ can be expressed in a compact form as,

$$
M\left(\omega_{1}, \omega_{2}\right)=H_{0} \frac{N_{R}-j N_{I}}{\left(D_{1 R}-j D_{1 I}\right) \cdot\left(D_{2 R}-j D_{2 I}\right)}
$$


where

$$
\begin{aligned}
& N_{R}=p_{00}+p_{00} f_{01}+p_{02} f_{02}+p_{10} f_{10}+p_{20} f_{20}+p_{11} f_{11}+p_{12} f_{12}+p_{21} f_{21}+p_{22} f_{22} \\
& N_{I}=p_{00}+p_{01001} g_{010202}+p_{10} p_{10010}+p_{20020} g_{20}+p_{16} g_{11}+p_{12} g_{12}+p_{21} g_{21}+p_{22} g_{22} \\
& D_{R}=1+q_{1} f_{10}+r_{1} f_{01}+s_{1} f_{11} \\
& D_{11}=1+q_{10} g_{10}+r_{1001} g_{11}+s_{1011} \\
& D_{2 R}=1+q_{2} f_{10}+r_{2} f_{01}+s_{2} f_{11} \\
& D_{2 l}=1+q_{2} g_{10}+r_{2001} g_{01}+s_{2} g_{11}
\end{aligned}
$$

with $f_{x y}=\cos \left(x \omega_{1}+y \omega_{2}\right)$

$$
g_{x y}=\sin \left(x \omega_{1}+y \omega_{2}\right)
$$

and $\mathrm{x}, \mathrm{y}=0,1,2$

Hence the actual magnitude may be written as,

$$
\left|M\left(\omega_{1}, \omega_{2}\right)\right|=H_{0} \sqrt{\frac{\left(N_{R}^{2}+N_{I}^{2}\right)}{\left(D_{1 R}^{2}+D_{1 I}^{2}\right)\left(D_{2 R}^{2}+D_{2 I}^{2}\right)}}
$$

Now let us consider a specific example of the design problem where the user specification for the desired circular symmetric low pass filter response may be given as,

$$
\begin{aligned}
\mathrm{M}_{\mathrm{d}}\left(\omega_{1}, \omega_{2}\right)=1, & \text { if } \sqrt{\omega_{1}^{2}+\omega_{2}^{2}} \leq 0.04 \pi \\
& =0.5, \quad \text { if } 0.04 \pi \leq \sqrt{\omega_{1}^{2}+\omega_{2}^{2}} \leq 0.08 \pi \\
& =0, \quad \text { otherwise. }
\end{aligned}
$$

Also from (4) the constraints may be put in a continuously differentiable form as,

$$
\begin{aligned}
& -\left(1+s_{k}\right)<\left(q_{k}+r_{k}\right)<\left(1+s_{k}\right) \\
& -\left(1-s_{k}\right)<\left(q_{k}-r_{k}\right)<\left(1-s_{k}\right) \\
& \left(1+s_{k}\right)>0 \\
& \left(1-s_{k}\right)>0
\end{aligned}
$$

Now in this example problem we select $\mathrm{b}=2, \mathrm{~N}_{1}=50$ and $\mathrm{N}_{2}=50$. Hence finally the constrained minimization task becomes,

Minimize $J$

$$
=\sum_{n_{1}=0}^{50} \sum_{n_{2}=0}^{50}\left[\left|M\left(\frac{\pi n_{1}}{50}, \frac{\pi n_{2}}{50}\right)\right|-M_{d}\left(\frac{\pi n_{1}}{50}, \frac{\pi n_{2}}{50}\right)\right]^{2}
$$

subject to constraints imposed by (15) with $\mathrm{k}=1,2$.

\subsection{Solution Vectors Representation and Initialization for DE}

In order to apply the DE-RANDSF algorithm to the problem formulated in (13) we need to represent each trial solution as a point in multi dimensional search space. Since $p_{00}$ is always set to 1 in (1) the dimensionality of the present problem is 14 and each particle has 14 positional coordinates represented by the vector,

$$
X=\left(p_{01}, p_{02}, p_{10}, p_{11}, p_{12}, p_{20}, p_{21}, p_{22}, q_{1}, q_{2}, r_{1}, r_{2}, s_{1}, s_{2}, H_{0}\right)^{T}
$$


Each component of a trial solution vector was initialized with a random floating point number whose absolute value was kept below 3.00.

\subsection{Handling the Constraints}

The constraint-handling method is following the criteria: a) any feasible solution is preferred to any infeasible solution; b) between two feasible solutions, the one having better objective function value is preferred; c) between two infeasible solutions, the one having smaller constraint violation is preferred. To tackle the constraints presented in (12) we start with a population of around 200 trial vectors with randomly initiated positions over the search space. Out of these, 40 vectors were selected, components of which obey the constraints imposed by (12). If more than 40 vectors were initially found to obey the constraints, obviously the selection will take into account the initial fitness value of these particles. During the run of the program, an offspring vector replaces its parent in the next generation only if besides yielding a better fitness value as compared to that of the parent it also satisfies the constraints. If the offspring vector is better than its parent but violates any of the constraints, the parent vector is retained in the next generation.

\subsection{GA Parameter Setup}

To illustrate the superiority of the proposed technique we also minimize the same fitness function given in (13) using a simple genetic algorithm as proposed in [5]. Chromosomes have the same parameters as shown in (15) and are initialized randomly. Each parameter or gene in a chromosome is converted into a 32 bit binary number. In each iteration, the chromosomes are subjected to usual crossover and mutation [6] operations and from the new population comprising parents and children, the members failing to satisfy the constraints given in (15) are deleted. From the remaining members, candidates for the next generation are selected according to their fitness value.

\section{Results of the Simulation and Comparison}

Fig. 1(a) below shows the desired amplitude response of the filter to be designed. Now in the present work 50 trials of the DE-RANDSF algorithm was run and the maximum permissible error limit was achieved within 400 iterations on average.

We took the average value of the best particle positions found in these 50 trials. The vector $\mathrm{X}$ found in this way is given by,

$$
\begin{aligned}
& X=[0.1596,1.2154,0.4302,0.5630,-0.3282,0.2889,-2.0931,1.7801,-0.9366, \\
& -0.9833,-0.8653,-0.8115,0.8551,0.8214,0.0005]^{T}
\end{aligned}
$$

The corresponding amplitude response is presented in Fig. 1(b). For the purpose of comparison we also present the amplitude response obtained by using the methods suggested in [4] and [5] in Fig. 1(c) and Fig. 1(d) respectively. A closer look at these figures reveals that the DE-RANDSF algorithm used by us yields a better 


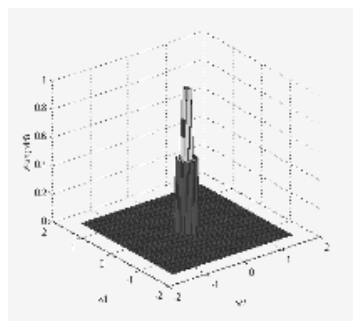

(a)

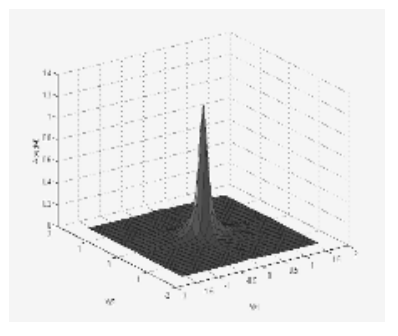

(b)

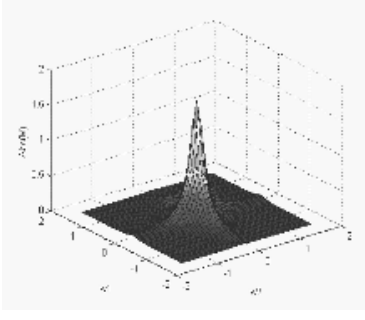

(c)

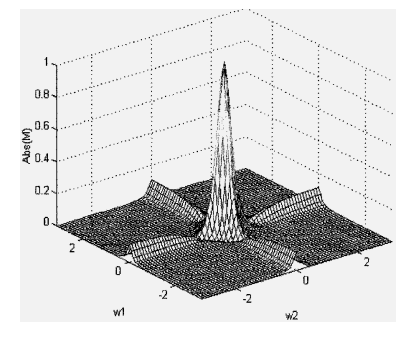

(d)

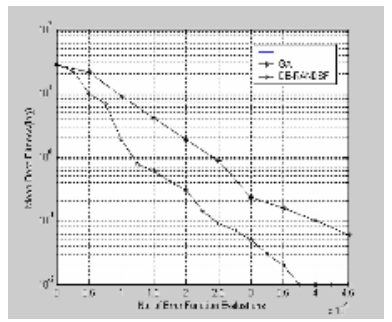

(e)

Fig. 1. (a) Desired amplitude response $\left|\mathbf{M}_{d}\left(\omega_{1}, \omega_{2}\right)\right|$ of the 2-D filter (b) Amplitude response $\left|\mathbf{M}\left(\omega_{1}, \omega_{2}\right)\right|$ of the 2-D filter using DE-RANDSF (c) Amplitude response $\left|\mathbf{M}\left(\omega_{1}, \omega_{2}\right)\right|$ of the 2D filter using GA (d) Amplitude response $\left|\mathrm{M}\left(\omega_{1}, \omega_{2}\right)\right|$ of the 2-D filter using the method in [5] (e) Performance Comparison for GA and DE-RANDSF algorithms

approximation of the desired response as compared to works presented in [4] or [5] and takes considerably smaller time for convergence. The ripple in the stop-band of Fig. 1(b) is much lesser as compared to Fig. 1(c) and Fig. 1(d).

\section{Conclusion}

In this work one of a newly proposed modified DE algorithm has been successfully applied to a practical optimization problem concerning the design of 2-D zero phase recursive filters. The filter thus obtained has a reasonably good stability margin. Our method also leads to a simpler filter since, in practice, we have to realize a factorable denominator and in particular of first-order filters. Compared to methods suggested in [4] and [5] the algorithm used here yields a much better design in a considerably small convergence time.

\section{References}

1. Kaczorek, T.: Two-Dimensional Linear Systems. Berlin, Germany: Springer-Verlag (1985)

2. Tzafestas, S, G.: Ed., Multidimensional Systems, Techniques and Applications. New York: Marcel Dekker (1986) 
3. Lu, W, S, Antoniou, A.: Two-Dimensional Digital Filters. NewYork: Marcel Dekker (1992)

4. Mladenov, V, Mastorakis, N.: Design of two-dimensional recursive filters by using neural networks, IEEE Trans. Neural Networks. Vol. 12. 585-590 (2001)

5. Mastorakis, N, Gonos, I, F, Swamy, M, N, S.: Design of two-dimensional recursive filters using genetic algorithms, IEEE Trans. Circuits and Systems. Vol. 50. 634-639 (2003)

6. Storn, R., Price, K.: Differential evolution - A Simple and Efficient Heuristic for Global Optimization over Continuous Spaces, Journal of Global Optimization, 11(4) (1997) 341-359.

7. Das, S., Konar, A., Chakraborty, U.K., Two improved differential evolution schemes for faster global search, to appear in the ACM-SIGEVO Proceedings of GECCO, Washington D.C., June 2005. 